\title{
Exchange bias-like magnetic properties in $\mathrm{Sr}_{2} \mathrm{LuRuO}_{6}$
}

\author{
R. P. Singh and C. V. Tomy \\ Department of Physics, Indian Institute of Technology Bombay, Mumbai-400076, India.
}

(Dated: July 2, 2021)

\begin{abstract}
Exchange bias properties are observed in a double perovskite compound, $\mathrm{Sr}_{2} \mathrm{LuRuO}_{6}$. The observed exchange bias properties have been analyzed on the basis of some of the available theoretical models. Detailed magnetization measurements show that the exchange bias properties are associated with the Dzyaloshinsky-Moria (D-M) interaction among the antiferromagnetically ordered Ru moments $\left(T_{N} \sim 32 \mathrm{~K}\right)$. In addition to the usual canting of the antiferromagnetic moments, D-M interaction in this compound also causes a magnetization reversal at $T \sim 26 \mathrm{~K}$, which seems to trigger the exchange bias properties. Heat capacity measurements confirm the two magnetic anomalies.
\end{abstract}

PACS numbers: 75.30.Et, 75.60.Jk, 75.50.Ee

\section{INTRODUCTION}

Exchange bias (EB) usually refers to an offset in magnetization hysteresis loop along the field axis 1 in an antiferromagnetic/ferromagnetic system due to the unidirectional anisotropy when the magnetization is measured after cooling down the sample in an external magnetic field below its magnetic ordering temperature. This effect is usually observed in some magnetic nanoparticles and thin films containing antiferromagnetic/ferromagnetic or ferromagnetic/spin-glass bilayers. However, this effect is also observed in some bulk materials such as manganites, cobaltates, intermetallic compounds ${ }^{2}$, spin-glass systems, etc. Exchange bias is of immense technological importance since it enables the control of reference magnetization in spintronic devices such as read-heads and nonvolatile memory. From a scientific point of view, it is of general interest because it involves a sophisticated interplay between fundamental magnetic properties such as anisotropy and exchange interaction as well as ferromagnetic and antiferromagnetic order. Despite the intensive research, especially in the last two decades, an understanding of the underlying microscopic coupling mechanism is still missing.

Very recently, Dong et. al. 10 has used Dzyaloshinsky-

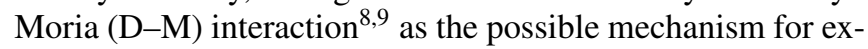
change bias in perovskites with compensated $G$-type antiferromagnetism. There are not many compounds which exhibit D-M interaction and hence detailed studies are not available regarding the EB like properties exhibited by them. We have found that the perovskite compound, $\mathrm{Sr}_{2} \mathrm{LuRuO}_{6}$, exhibits exchange bias-like properties from our magnetization measurements. The D-M interaction generally occurs when the crystal structure has low symmetry. The family of double perovskite compounds $\mathrm{Sr}_{2} L n \mathrm{RuO}_{6}$ (where $L n=\mathrm{Y}$ or rare earth) form in a monoclinic structure (space group $\left.P 2_{1} / n\right)^{\sqrt{12}}$ and are good candidates for exhibiting D-M interaction ${ }^{13 \mid 14}$ due to distortion of the oxygen octahdra ${ }^{15}$. Since there are no reports available for the detailed magnetic properties of $\mathrm{Sr}_{2} \mathrm{LuRuO}_{6}$, we first give the detailed magnetic properties of this compound, which is followed by the exchange bias studies. Heat capacity measurements confirm the two anomalies, corresponding to the two magnetic orderings. Our results further show that this compound shows exchange bias properties associated with the D-M interaction.

\section{EXPERIMENTAL DETAILS}

Polycrystalline sample of $\mathrm{Sr}_{2} \mathrm{LuRuO}_{6}$ was prepared by the standard solid state reaction method. Stoichiometric amounts of $\mathrm{SrCO}_{3}, \mathrm{Lu}_{2} \mathrm{O}_{3}$ and $\mathrm{Ru}$ metal powder were mixed thoroughly and heated initially at $960^{\circ} \mathrm{C}$ for 24 hours. The samples were then given two more intermediate heat treatments at $1350^{\circ} \mathrm{C}$, before pelletizing and sintering at $1360^{\circ} \mathrm{C}$ for 24 hours. Diffraction pattern of the sample was recorded on an X'pert PRO X-ray diffractometer (PANalytical, Holland). The Rietveld analyses of the X-ray diffraction patterns using FULLPROF software showed that the compound forms in a single phase. The observed patterns could be indexed to a monoclinic structure with a space group $P 2_{1} / n$. The lattice parameters obtained from the analyses are $a=5.727(2) \AA, b$ $=5.727(2) \AA$ and $c=8.101(3) \AA$ along with $\beta=90.2^{\circ}$, which are in good agreement with those values reported earlier ${ }^{16}$. The magnetization as a function of temperature and magnetic field was measured using a vibrating sample magnetometer (VSM) attachment of the Physical Property Measurement System (PPMS) (Quantum Design, USA).

\section{RESULTS AND DISCUSSION}

\section{A. Magnetic properties}

Figure 1 illustrates the magnetization of $\mathrm{Sr}_{2} \mathrm{LuRuO}_{6}$ as a function of temperature in zero field-cooled (ZFC) and fieldcooled (FC) modes. For low field values, the ZFC magnetization is negative at lower temperatures. As the temperature is increased, the magnetization decreases to go through a minimum, which is contrary to the normal behaviour. As the temperature is further increased, the magnetization increases, goes through a positive maximum, and then shows the normal paramagnetic behaviour at high temperatures (not shown). For higher fields ( $\geq 500 \mathrm{Oe}$ ), the magnetization is positive at all temperatures. As shown in Fig. 1(b), the FC magnetisation is positive at all temperatures, shows a broad peak centred around $T \sim 26 \mathrm{~K}$ and the temperature at which the peak occurs shows a weak temperature dependence on the applied fields. The nature of increase in FC magnetiza- 


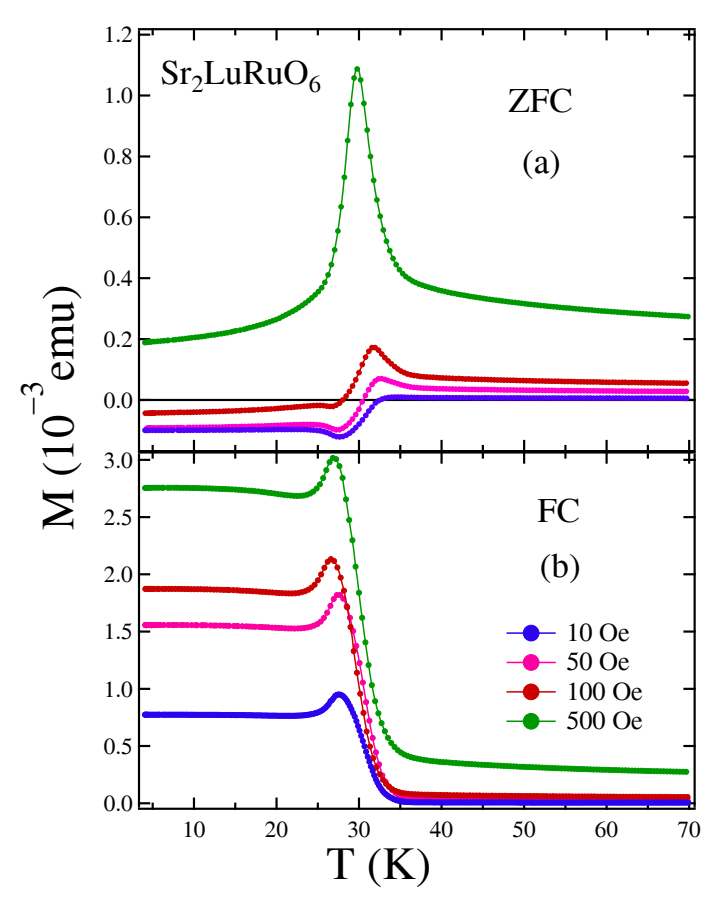

FIG. 1: Magnetization vs temperature for $\mathrm{Sr}_{2} \mathrm{LuRuO}_{6}$ in (a) zero field-cooled (ZFC) and (b) field-cooled (FC) modes under various applied fields.

tion near $T_{N} \sim 32 \mathrm{~K}$ clearly indicates the presence of a ferromagnetic component which is expected in this compound due to D-M interaction among the antiferromagnetically ordered $\mathrm{Ru}$ moments. In order to confirm the presence of ferromagnetic component as well as to find the origin of the anomalous behaviour in magnetization, we have measured hysteresis loops at different temperatures. Typical hysteresis loops are shown in Fig. 2(a)-(h) for selected temperatures. At low temperatures, the hysteresis is negligible and the loops are almost closed. Above $T \sim 20 \mathrm{~K}$, the hysteresis loops open up and the coercivity increases as shown in Fig. 2(i). Above $T \sim 26 \mathrm{~K}$, the hysteresis loops start closing again, the coercivity decreases and disappears above the magnetic ordering temperature of $T_{N} \sim 32 \mathrm{~K}$. As the sample is cooled through $T_{N}$, the increase in coercivity can be attributed to the ferromagnetic component developed due to the $\mathrm{D}-\mathrm{M}$ interaction. The decrease in coercivity below $T \sim 26 \mathrm{~K}$ implies a net decrease in the ferromagnetic component. The absence or negligible coercivity below $20 \mathrm{~K}$ can result from the disappearance of the ferromagnetic component. However, this possibility is quite unlikely since no structural change occurs in this compound ${ }^{16}$ and hence the D-M interaction (which gives rise to canting) cannot vanish. Another possibility is the magnetization reversal by which some of the Ru moments re-align opposite to the ordered $\mathrm{Ru}$ moments reducing/cancelling the net ferromagnetic component. This will explain the ZFC/FC magnetization behaviour also. When the sample is cooled below $T_{N}$, the FM component increases the magnetization in FC measurements. At $T=26 \mathrm{~K}$, the reversal occurs, some moments align opposite to the field and the magnetization decreases. Since all

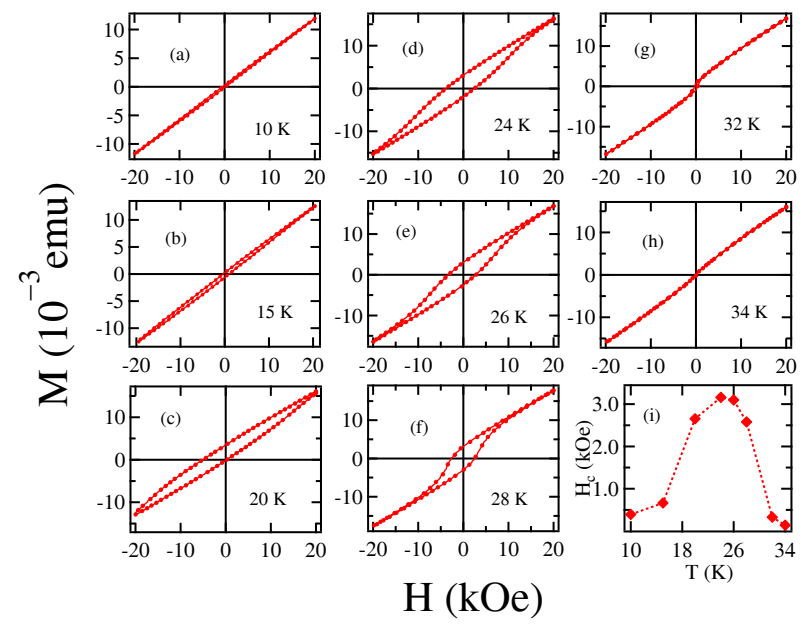

FIG. 2: Isothermal magnetization curves (a)-(h) obtained under ZFC mode for $\mathrm{Sr}_{2} \mathrm{LuRuO}_{6}$ at different temperatures. (i) Coercivity $\left(H_{\mathrm{c}}\right)$ as a function of temperature obtained from the same curves.

the moments do not reverse/re-align, the magnetization does not go to zero, but decreases and remains constant when the reversal is complete. In ZFC measurements, even though the applied field is zero, a small amount of negative remnant field can orient the moments along the negative field direction at $T_{N}$ and some of the moments re-align along the positive direction at the reversal temperature, resulting in a mirror image of the FC curves. At the lowest temperature, when the positive applied field is small, moments does not change the status and hence the magnetization is negative. As the temperature is increased to the magnetization reversal temperature, moments aligned along the positive direction (those reversed), reverse back to the negative direction, resulting in a sudden increase in the negative magnetization. As the temperature is further increased, all the moments have to align along the positive field direction when $T_{N}$ is approached, resulting in a positive maximum.

Heat capacity measurements were carried out in the temperature range $2-200 \mathrm{~K}$. Figure 3(a) shows the measured heat capacity. Two anomalies can be noted around the magnetic ordering temperatures (see the expanded portion near the magnetic ordering in the inset of Fig. 3(a)). In order to obtain the magnetic contribution to heat capacity, the phonon contribution needs to be separated from the total heat capacity. As there is no nonmagnetic analogue available for this compound, the total heat capacity above the magnetic ordering temperature was fitted to an equation containing standard Einstein and Debye terms $\frac{17}{17}$,

$$
C_{\mathrm{ph}}=R\left(\frac{1}{1-\alpha_{D}}\left(\frac{\theta_{D}}{T}\right)^{3} \int_{0}^{x} \frac{x^{4} e^{x}}{\left(e^{x}-1\right)^{2}} d x+\sum_{i=1}^{3 n-n} \frac{1}{1-\alpha_{E}} \frac{y^{2} e^{y}}{\left(e^{y}-1\right)^{2}}\right)
$$

where $\alpha_{E}$ and $\alpha_{D}$ are the anharmonicity coefficients, $\theta_{D}$ is the Debye temperature, $\theta_{E}$ is the Einstein temperature, $x=\theta_{D} / T$ 

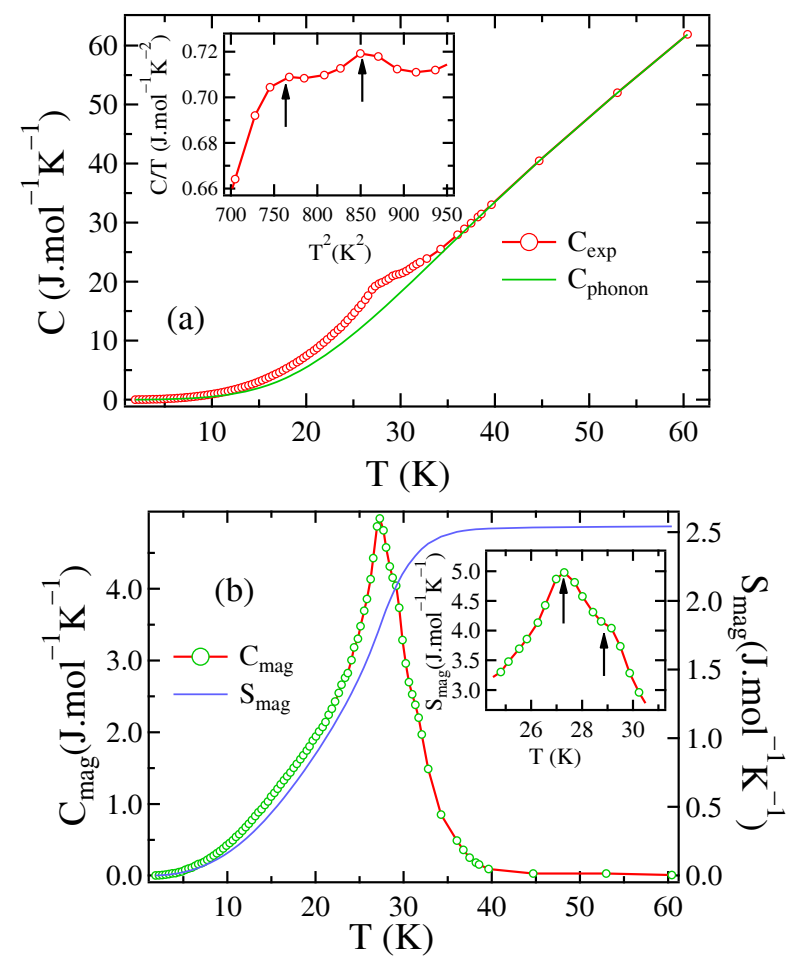

FIG. 3: Total measured heat capacity (circles) as a function of temperature. The inset shows the expanded portion near the magnetic ordering temperatures. Arrows indicate the two magnetic anomalies. Solid line is the calculated phonon contribution. (b) left scale : magnetic contribution to heat capacity $\left(C_{\mathrm{mag}}\right)$ obtained by subtracting the calculated phonon contribution from the measured heat capacity as a function of temperature. Inset shows the expanded portion near the magnetic anomalies. The magnetic entropy $\left(S_{\mathrm{mag}}\right)$ is plotted on the right scale.

and $y=\theta_{E i} / T$. The best possible fit was obtained when the calculations were performed by using one Debye and three Einstein frequencies along with a single $\alpha_{E}$. The solid line in Fig. 3(a) represents the fit to the phonon contribution (extended over the whole temperature range), which is in good agreement with the experimental data at high temperatures (above the magnetic ordering). The parameters obtained from the best fit are: $\theta_{D}=228 \mathrm{~K}, \theta_{E 1}=130 \mathrm{~K}, \theta_{E 2}=507 \mathrm{~K}$, $\theta_{E 3}=510 \mathrm{~K}, \alpha_{E}=9.0 \times 10^{-4} \mathrm{~K}^{-1}$ and $\alpha_{D}=9.5 \times 10^{-5} \mathrm{~K}^{-1}$. The calculated phonon contribution was then subtracted to obtain the magnetic heat capacity, which is shown in Fig. 3(b). The well-defined peak indicates the presence of a clear long range magnetic ordering. However, a closer look can reveal a shoulder, indicated by the arrow, consistent with two transitions in the magnetization measurements. The slight broadening below the magnetic ordering may be caused by the subtraction of the extrapolated phonon contribution, which was calculated only above the magnetic ordering temperatures. The magnetic entropy calculated from the magnetic heat capacity $\left(S_{\operatorname{mag}} \sim 2.56 \mathrm{~J} \mathrm{~mol}^{-1} \mathrm{~K}^{-1}\right)$ is also found to be well below the expected value even for a doublet ground state of $\mathrm{Ru}\left(S_{\mathrm{mag}} \sim 5.76 \mathrm{~J} \mathrm{~mol}^{-1} \mathrm{~K}^{-1}\right)$, consistent with the findings in other members of this family 18119 . Our preliminary results from inelastic neutron scattering measurements ${ }^{\sqrt{20}}$ have indicated the presence of crystal field levels for $\mathrm{Ru}$ in $\mathrm{Sr}_{2} \mathrm{YRuO}_{6}$. This crystalline field effect can reduce the ground state of $\mathrm{Ru}^{5+}$ to a doublet ground state. Further, we have observed ${ }^{20121}$ the presence of diffuse scattering even well above the magnetic ordering temperature. This diffuse scattering is expected from the frustration among the Ru moments. If frustration exists among the magnetic moments, it can reduce the entropy of spins while entering the magnetically ordered state. If we consider similar mechanisms operating in $\mathrm{Sr}_{2} \mathrm{LuRuO}_{6}$ also, then the decrease in magnetic entropy in the present case can be attributed to the crystalline electric field effects and frustration among the Ru moments.

\section{B. Exchange bias-like properties}

In order to further verify the detailed magnetic behaviour, we have measured the exchange bias properties of this compound. One of the methods to verify the presence of exchange bias is to measure the hysteresis loop after field-cooling (FC) the sample below its $T_{N}$, and compare it with the hysteresis loop obtained in ZFC mode. For the FC process, the sample was cooled to $20 \mathrm{~K}$ in a magnetic field of $50 \mathrm{kOe}$ (in zero applied field for ZFC process) and the hysteresis loops were then measured between $\pm 90 \mathrm{kOe}$, as shown in Fig. 4(a). A clear

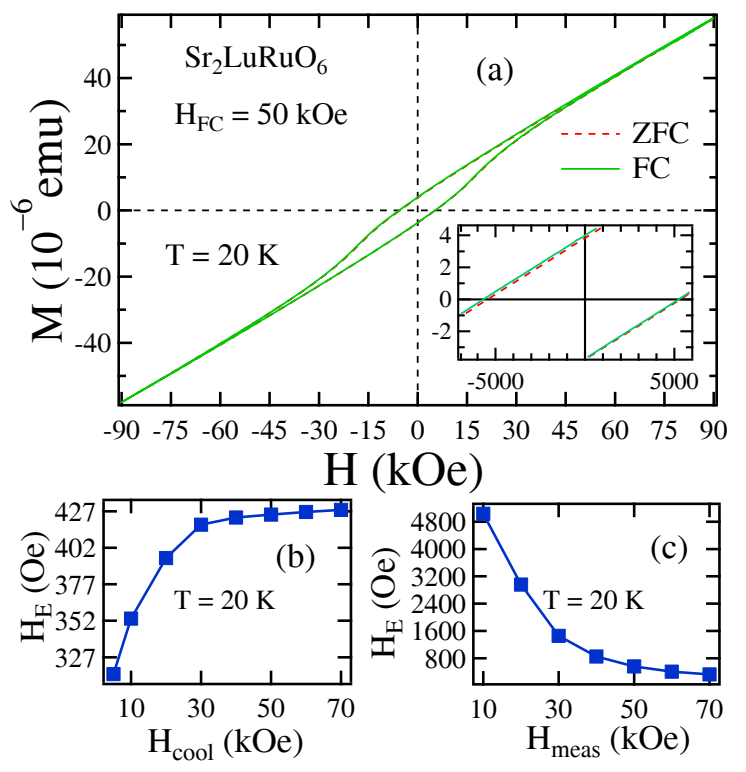

FIG. 4: (a) Expanded portion of magnetization hysteresis $(M-H)$ loops near origin for $\mathrm{Sr}_{2} \mathrm{LuRuO}_{6}$ at $20 \mathrm{~K}$ measured in zero fieldcooled (ZFC) and field-cooled (FC) modes. For FC mode, the cooling field $\left(H_{\mathrm{FC}}\right)$ is $50 \mathrm{kOe}$. (b) Exchange bias $(\mathrm{EB})$ field $\left(H_{E}\right)$ values deduced from $\mathrm{FC}$ hysteresis loops as a function of measuring field, $H_{\text {meas }}$. (c) Cooling field dependence of $H_{E}$ where the EB field values are plotted as a function of cooling field $\left(H_{\mathrm{FC}}\right)$. 


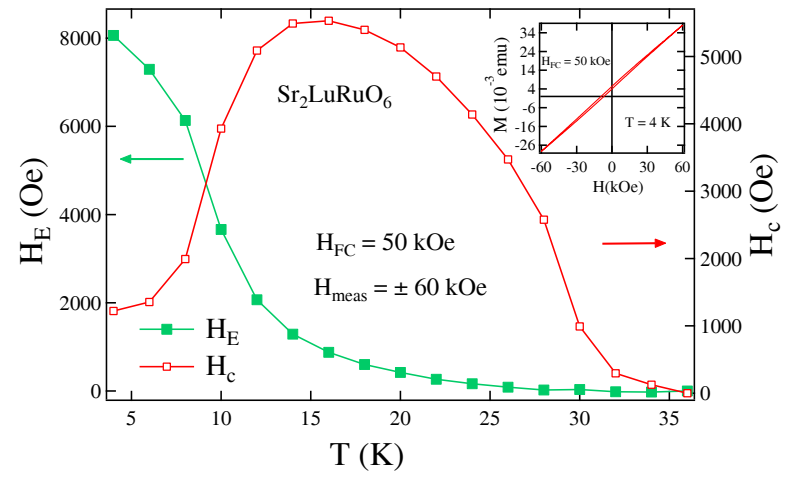

FIG. 5: (left scale): Temperature variation of the EB field $H_{\mathrm{E}}$. The $H_{\mathrm{E}}$ values were deduced from the $\mathrm{FC}$ hysteresis loops obtained at each temperature. (right scale): Temperature variation of coercivity $H_{\mathrm{c}}$ obtained from the same hysteresis loops. Inset shows a typical hysteresis loop at low temperatures $(4 \mathrm{~K})$, showing negligible coercivity, but large offset causing large $H_{\mathrm{E}}$ values.

difference between the two hysteresis loops can be resolved (see the inset of Fig. 4(a) where the expanded portion near the origin is plotted); while the ZFC hysteresis loop is centred at zero field, the FC hysteresis loop shifts towards both the negative field and the positive magnetization direction. This shift was further confirmed by measuring the FC hysteresis in a cooling field of $-50 \mathrm{kOe}$, which showed the shift along positive field and negative magnetization direction. We define the shift along the field axis as the exchange-bias field $H_{E}$ $\left(=-\left(H_{c+}+H_{c-}\right) / 2\right)^{22}$, where $H_{c+}\left(H_{c-}\right)$ is the coercive field on the right (left) side of the zero field. The observed value of $H_{E}$ is $216 \mathrm{Oe}$, indicating the presence of an exchange field in this compound. In EB measurements, cooling field is known to affect the exchange bias. In order to select a suitable cooling field, we measured the $M-H$ loops in various cooling fields ranging from $5 \mathrm{kOe}$ to $70 \mathrm{kOe}$ at $20 \mathrm{~K}$. The exchange field values calculated from the loop shift is shown in Fig. 4(b). The exchange bias field shows a saturation type behaviour above $30 \mathrm{kOe}$. Another factor affecting the exchange bias measurement is the maximum value of the field to which the field is cycled (minor hysteresis loop effects). To circumvent this effect and select a suitable measuring field, we measured hysteresis loops between different field values after field-cooling the sample in a field of $50 \mathrm{kOe}$. Loops obtained at low cycling fields show large exchange bias values (see Fig. 4(c)) due to the fact that the loops are not closed or not reached the saturation (minor hysteresis). However, as the cycling field exceeds $\pm 30 \mathrm{kOe}$, the loops start closing and the exchange field tends to saturate. Since we have used $H_{\mathrm{FC}}=50 \mathrm{kOe}$ and a measuring field of $60 \mathrm{kOe}$, we can ascertain that the exchange field obtained in our measurements is the inherent property of the sample and not an artefact of the measuring field or cooling field 23 .

In order to verify whether the observed EB like properties are associated with the magnetic ordering, we studied the temperature dependence of exchange bias. In these measurements, the sample was field-cooled to the measuring temper- ature in an applied field of $50 \mathrm{kOe}$. Once the measuring temperature was reached, the magnetization loops were measured between $\pm 60 \mathrm{kOe}$. Figure 5 shows variation of the exchange field $H_{E}$ at different temperatures, along with a plot of coercivity $H_{c}$. At $4 \mathrm{~K}$, the exchange field is as high as 8000 Oe. This happens due to a large offset of the hysteresis loop along the magnetization/field axis (see inset of Fig. 5). As the temperature is increased, $H_{E}$ value falls sharply and almost disappears around $26 \mathrm{~K}$, even though coercivity and hence FM component persists upto $32 \mathrm{~K}$, which is the magnetic ordering temperature for this compound. The sudden increase of exchange field at low temperatures indicates that the origin of exchange bias properties in this compound is not purely from the appearance of the FM component (and hence D-M interaction). It is more associated with the second magnetic anomaly at $T \sim 26 \mathrm{~K}$, which reverses the magnetization.

We have measured another important property called the training effect to ascertain the exchange bias in our compound, which is described as the decrease of the exchange bias when the systems is cycled through several successive hysteresis loops. Inset of Fig. 6 shows the expanded portion of the lowfield region in the negative field quadrant. The arrow indicates the direction of increase in the field cycle $(n)$. The curves in the positive field quadrant do not show any visible change. It is clear that the training effect is present in our sample and the exchange bias decreases as the number of cycles is increased, as shown in the main panel of Fig. 6, where the exchange bias field is plotted as a function of $n$, the number of times through which the sample is cycled in the field. The dependence of exchange-bias on the number of field cycles $(n)$ is usually given by a simple power-law relationship (for $n>1)^{\sqrt{2}}$.

$$
H_{E}(n)-H_{E \infty}=k / \sqrt{n}
$$

Here $H_{E}(n)$ is the exchange field at the $n^{\text {th }}$ cycle, $H_{E \infty}$ is the exchange field after a large number of field cycling $(n \rightarrow \infty)$ and $k$ is a system dependent constant. The solid line in Fig. 6 represents the best fit with the above empirical relation. We have obtained $H_{E \infty}=170 \mathrm{Oe}$, which will be the remnant exchange bias field in the sample. Since this power-law breaks down for $n=1$, another approach to explain training effect is given by Binek ${ }^{26}$, where the training effect in FM/AFM heterostructures is analyzed in the framework of nonequilibrium thermodynamics. It was proposed that the FM top layer, when consecutively cycled through magnetic fields, triggers a spin configurational relaxation of the AFM interface magnetization toward equilibrium. With this consideration, a recursive formula, instead of the power-law formula, is given to describe the field cycling dependence of exchange bias as,

$$
H_{E}(n+1)-H_{E}(n)=-\gamma\left(H_{E}(n)-H_{E \infty}\right)^{3}
$$

where $\gamma$ is again a sample dependent constant $\left(=1 / 2 k^{2}\right)$. We have attempted to generate data with Eq. (3) using $\gamma=$ $4.1 \times 10^{-5} \mathrm{Oe}^{-2}$ and $H_{E \infty}=170 \mathrm{Oe}$ (obtained from the power law fit, Eq. (2)) as additional inputs. The open (green) circles in Fig. 6 represent the $H_{E}$ values generated by Eq. (3), which match satisfactorily with the experimental data. Thus the spin 


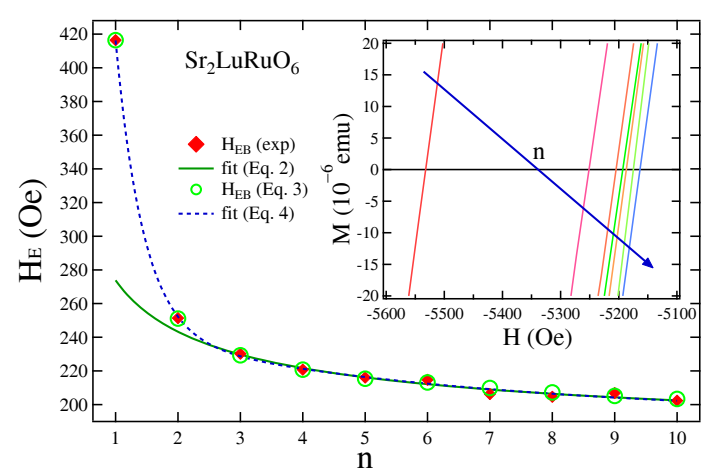

FIG. 6: The EB field $\left(H_{\mathrm{E}}\right)$ dependence on the number of field cycles (n) (red diamonds) obtained from the training effect hysteresis loops. The solid line represents the best fit for $H_{\mathrm{E}}$ data to Eq. 22 for $n>1$. Open circles (green) are the data points generated using the recursive formula, Eq. 3). The dotted blue line shows the best fit with Eq. (4). Inset shows part of the hysteresis loops, demonstrating the training effect due to exchange bias in $\mathrm{Sr}_{2} \mathrm{LuRuO}_{6}$. Arrow indicates the direction of increase in field cycle $(n)$.

configurational relaxation model can describe our experimental results as well, which prima-facie is not a multi-layer compound. It is likely that the consecutive reversing of the FM component (generated by $\mathrm{D}-\mathrm{M}$ interaction) triggers the configurational relaxation of the interfacial $\mathrm{AF}$ spins toward equilibrium and causes the training effect.

Recently Mishra et. al ${ }^{28}$ considered the training effect as related to the interfacial spin disorder. The evolution of the interfacial disorder during the measurements of the hysteresis loops causes a decrease of the exchange bias field. AF domain dynamics is also found to affect the magnitude of coercive as well as exchange bias fields. The combined effect of these contributions causes a gradual decrease of exchange bias as a function of $n$. The exchange bias then can be given by a probabilistic equation,

$$
H_{E}(n)=H_{E \infty}+A_{f} e^{-n / P_{f}}+A_{i} e^{-n / P_{i}}
$$

where $A_{f}$ and $P_{f}$ are parameters related to the change of the frozen spins, $A_{i}$ and $P_{i}$ are parameters related to the evolution of the interfacial disorder. The $A$ parameters have dimension of field while the $P$ parameters have no dimension but they are similar to a relaxation time, where the continuous variable "time" is replaced by a discrete variable $n$. We have used this equation to fit our experimental data, which is shown as the dotted (blue) line in Fig. 6, which agrees well with the experimental data. The parameters obtained from this fit are: $H_{E \infty}=192 \mathrm{Oe}, A_{f}=55.8 \mathrm{Oe}, P_{f}=5.5, A_{i}=1628 \mathrm{Oe}$, $P_{i}=0.45$. The value of $H_{E \infty}=192$ Oe compares well with the value $\left(H_{E \infty}=170 \mathrm{Oe}\right)$ obtained from the power-law fit (Eq. (2)). A comparison between $P_{f}$ and $P_{i}$ shows that the frozen component relaxes nearly 10 times faster than the interfacial magnetic frustration.

\section{CONCLUSION}

We have shown that the perovskite compound, $\mathrm{Sr}_{2} \mathrm{LuRuO}_{6}$, exhibits properties associated with exchange bias. The observed exchange bias properties could be analyzed on the basis of some of the available theoretical models. The D-M interaction can be considered as the possible mechanism behind the anomalous magnetic behaviour shown by this compound. However, the indications are that the exchange bias properties become dominant only after the magnetization reversal, and not along with the magnetic order with FM component. Detailed measurements are needed to pinpoint the exact mechanism for the complex magnetic behaviour in this compound. In short, out results support the proposal by Dong et. al. that the perovskites with compensated AFM structure and weak ferromagnetic component (coming from D-M interactions) can show the exchange bias like properties.
1 W. H. Meiklejohn and C. P. Bean, Phys. Rev. 102 (1956) 1413.

2 Nogus and I. K. Schuller, J. Magn. Magn. Mater. 192 (1999) 203.

3 W. H. Meiklejohn, J. Appl. Phys. 33 (1962) 132.

${ }^{4}$ L. Nel, Ann. Phys. 2 (1967) 61

5 D. Mauri, H. C. Siegmann, P.S. Bagus and E. Kay, Appl. Phys. 62 (1987) 3047.

6 A. P. Malozemoff, Phys. Rev. B 35 (1987) 3679.

7 N. C. Koon, Phys. Rev. Lett. 78 (1997) 4865.

8 I. Dzyaloshinsky, J. Phys. Chem. Solids 4 (1958) 241.

9 T. Moriya, Phys. Rev. 120 (1960) 91.

${ }^{10}$ S. Dong, K. Yamauchi, S. Yunoki, R .Yu, S. Liang, A. Moreo, J-M Liu, S. Picozzi and E. Dagotto, Phys. Rev. Lett. 130 (2009) 127201.

11 P. C. Donohue and E. L. McCann, Mater. Res. Bull. 12 (1977) 519.

12 P. D. Battle and W. J. Macklin, J. Solid State Chem. 54245.

13 R. Greatrex, N. N. Greenwood, M. Lal and I. Fernandez, J. Solid State Chem. 30 (1979) 137.

14 Y. Doi and Y. Hinatsu, J. Phys.: Condens. Matter, 11 (Y 1999) 4813.

15 S. G. Ovchinnikov, Phys.-Usp. 46 (2003) 21.
16 P. D. Battle and C. W. Jones, J. Solid State Chem 78 (1989) 108.

17 C. A. Martin, J. Phys.: Condens. Matter 3 (1991) 5967.

18 R. P. Singh and C. V. Tomy, Phys. Rev. B 77 ( 2008) 024432.

19 R. P. Singh and C. V. Tomy, J. Phys.: Condens. Matter 20 (2008) 235209.

20 ISIS Experimental Report RB Number: 810077.

${ }^{21}$ ISIS Experimental Report RB Number: 810073.

22 D. Niebieskikwiat and M. B. Salamon, Phys. Rev. B 72 (2005) 174422.

23 J. Geshev, J. Magn. Magn. Mater. 320 (2008) 60.

${ }^{24}$ G. Salazar-Alvarez, J. Sort, S. Suriach, M. D. Bar and J. Nogus, J. Am. Chem. Soc. 129 (2007) 9102.

25 C. Leighton, H. Suhl, M. J. Pechan, R. Compton, Nogus and I. K. Schuller, J.Appl. Phys 93 (2002) 1483.

26 C. Binek, Phys. Rev. B 70 (2004) 014421.

27 A. Hochstrat, C. Binek and W. Kleemann, Phys. Rev. B 66 (2002) 092409.

28 S. K. Mishra, F. Radu, H. A. Drr and E. Eberhardt, Phys. Rev. Lett. 102 ( 2009) 177208. 